\title{
Targeting a genetic defect: cystic fibrosis transmembrane conductance regulator
} modulators in cystic fibrosis

\author{
Nico Derichs
}

ABSTRACT: Cystic fibrosis (CF) is caused by genetic mutations that affect the cystic fibrosis transmembrane conductance regulator (CFTR) protein. These mutations can impact the synthesis and transfer of the CFTR protein to the apical membrane of epithelial cells, as well as influencing the gating or conductance of chloride and bicarbonate ions through the channel. CFTR dysfunction results in ionic imbalance of epithelial secretions in several organ systems, such as the pancreas, gastrointestinal tract, liver and the respiratory system. Since discovery of the CFTR gene in 1989, research has focussed on targeting the underlying genetic defect to identify a disease-modifying treatment for CF. Investigated management strategies have included gene therapy and the development of small molecules that target CFTR mutations, known as CFTR modulators. CFTR modulators are typically identified by high-throughput screening assays, followed by preclinical validation using cell culture systems. Recently, one such modulator, the CFTR potentiator ivacaftor, was approved as an oral therapy for CF patients with the G551D-CFTR mutation. The clinical development of ivacaftor not only represents a breakthrough in CF care but also serves as a noteworthy example of personalised medicine.

KEYWORDS: CFTR modulators, cystic fibrosis, G551D mutation, ivacaftor, personalised medicine, VX-770

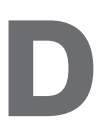

orothy Hansine Andersen was the first person to comprehensively describe and document cystic fibrosis (CF) in the 1930s [1]. In subsequent decades, clinical practice evolved in a stepwise fashion by delivering improved symptom-modifying treatments that improved patient prognosis and quality of life. This was mainly achieved by nutritional supplementation, physiotherapy and administration of antibiotic/ anti-inflammatory medication [2]. Research into the underlying pathophysiology, however, made a significant leap forward with the identification of the underlying cause of $\mathrm{CF}$, mutations in the cystic fibrosis transmembrane conductance regulator (CFTR) gene [3-5]. The advance of genomics-led research provided a sound basis to identify novel drug candidates that target and repair specific CFTR mutations and, in doing so, ushered CF care into a new era of personalised medicine [6].

\section{GENETICS OF CFTR}

$\mathrm{CF}$ is a rare monogenic disorder that mainly affects individuals of Caucasian descent. In Europe, around one in every 2,000-3,000 neonates is diagnosed with CF and in the USA this incidence is reported to be slightly less at one in 3,500 births $[7,8]$. In order to manifest $\mathrm{CF}$, an individual must inherit two defective CFTR genes (or alleles), one from each parent. Promotion of the CFTR gene normally results in expression of the CFTR protein: a transmembrane channel located in the apical membrane of epithelial cells responsible for the transport of chloride and bicarbonate ions into and out of the cell.

The CFTR gene is located on the long arm of chromosome 7 at position q31.2 [9]. To date, over 1,900 sequence variations in the CFTR gene have been reported [10], although a detailed understanding of how CFTR mutations impact channel dysfunction is limited to a only a few of these [11]. This implies a relatively small list of welldefined CFTR mutations known to be CF disease causing, whereas the functional consequence of various rare sequence variations remains unknown or has been shown not to be CF disease
AFFILIATION

CFTR Biomarker Centre and Translational CF Research Group Christiane Herzog Cystic Fibrosis Centre, Paediatric Pulmonology and Immunology, Charité

Universitätsmedizin Berlin, Berlin, Germany.

CORRESPONDENCE

N. Derichs

CFTR Biomarker Centre and

Translational CF Research Group Christiane Herzog Cystic Fibrosis Centre

Paediatric Pulmonology and

Immunology

Charité Universitätsmedizin Berlin Augustenburger Platz 1

13353 Berlin

Germany

E-mail: Nico.Derichs@charite.de

Received:

Dec 202012

Accepted after revision:

Jan 122013

PROVENANCE

Publication of this peer-reviewed article was supported by Vertex Pharmaceuticals Inc., USA (principal sponsor, European Respiratory Review issue 127).

European Respiratory Review Print ISSN 0905-9180 Online ISSN 1600-0617 
causing [12]. The majority of identified CFTR gene mutations fall into one of six classes that account for $\sim 80 \%$ of all $C F$ patients [9]. Of the $>1,900$ CFTR mutations that have been identified so far, approximately only 20 mutations have a frequency $>0.1 \%$ [9]. In fact, notwithstanding the common F508del variant, only four CFTR mutations have a frequency $>0.1 \%$ : G551D, W1282X, G542X and N1303K, having a worldwide prevalence of around $1-3 \%$ each $[9,11]$. F508del is the most common mutation with an allelic frequency of around $90 \%$ worldwide [13].

\section{Mutation classification}

Class I and II CFTR mutations are typically characterised by a reduction in the quantity of expressed CFTR protein (fig. 1). Class I mutations can result from nonsense and frame-shift mutations, as well as mRNA splicing defects [14]. For example, G542X is a nonsense or stop mutation, where introduction of a premature termination codon (or stop codon) results in premature cessation of translation and production of truncated CFTR protein. Class II mutations, including F508del, have folding or maturation defects, which can result in premature CFTR degradation (fig. 1) [9].

Class III and IV mutations, however, are typified by aberrant channel function rather than reduced quantities of CFTR. Class III mutations result in limited channel gating that arises from ineffectual binding of nucleotide; an example is G551D which accounts for $2-3 \%$ of CFTR mutations worldwide. CFTR channels with Class IV mutations are able to open and close; however, chloride and bicarbonate ions are unable to freely pass through the channel due to conductance defects [9].

While Class V mutations can lead to the production of normal CFTR, a limitation of transcriptional regulation results in a reduced quantity of the protein being produced $[9,11]$. Finally, the relatively novel Class VI mutations are characterised by high turnover of CFTR at the channel surface (fig. 1) [15].

\section{CFTR PROTEIN STRUCTURE}

The CFTR protein is a cyclic adenosine monophosphateregulated chloride ion channel composed of 1,480 amino acids [16]. The channel is an ATP-binding cassette transporter and is composed of five domains: two transmembrane domains (TMD1/TMD2) that form the channel pore, one regulatory domain (R), and two nucleotide binding domains (NBD1/ NBD2) [17]. Classical channel opening is thought to be brought about by phosphorylation of the $\mathrm{R}$ domain by protein kinase $\mathrm{A}$ and recruitment of ATP to the nucleotide binding domains, NBD1 and NBD2. These domains dimerise (NBD1:NBD2) to open the channel pore. Upon subsequent ATP hydrolysis, the

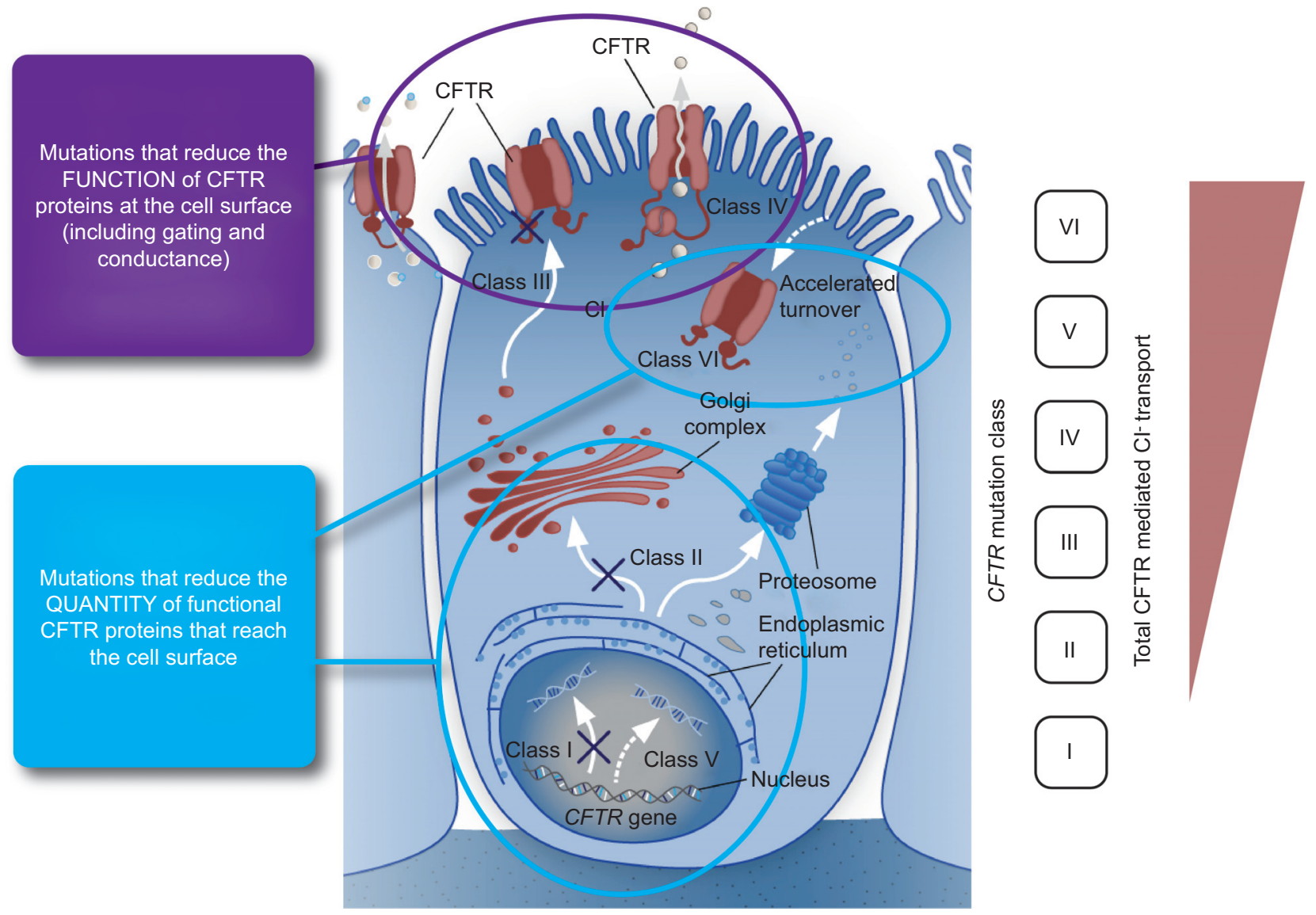

FIGURE 1. Cystic fibrosis transmembrane conductance regulator (CFTR) gene mutations are categorised into six classes. Mutation classes I, II, V and VI result in an absence or reduced quantity of CFTR protein at the cell membrane, whereas mutation classes III and IV influence the function or activity of CFTR at the cell membrane. Class I mutations are associated with the greatest disruption to CFTR-mediated chloride transport; in general, chloride transport gradually increases through the remaining five classes, with the greatest activity being observed in Class IV-VI mutations. 
NBDs disassociate to close the channel $[9,17]$. The entire process of channel opening and closing is aptly termed channel "gating".

\section{CFTR PROTEIN SYNTHESIS}

Under normal circumstances, extracellular signals instigate CFTR gene expression by promoting transcription of the CFTR gene into mRNA. The resultant single strand template then migrates through nuclear pores to interact with ribosomes in the cytoplasm or in the endoplasmic reticulum. Here, in combination with transfer RNA, the CFTR gene is translated into nascent chains of amino acids $[9,16]$. The resulting polypeptides are assembled into the immature CFTR protein product, which is subsequently folded within the lipid bilayer of the endoplasmic reticulum [18]. Following further maturation steps in the endoplasmic reticulum, CFTR protein is then transferred to the Golgi apparatus for post-translational modification and packaging into transport vesicles. Finally, the channel is transported or "trafficked" to the cell surface for final expression on the apical membrane of epithelial cells $[9,16]$. The entire process is summarised in figure 1. Mutations in the CFTR gene can impact each step in protein synthesis: from gene transcription, through protein translation, folding and trafficking, to expression and gating of the channel on the cell surface.

\section{CLINICAL MANIFESTATIONS OF CF}

The clinical manifestations of CF have been noted in several organ systems with the respiratory system being the worse affected. With the exception of the sweat gland, regardless of organ or tissue, the absence or dysfunction of CFTR results in an ionic imbalance that results in the secretion of thick, dehydrated mucus [19]. Mucosal obstruction of exocrine glands is the main cause of CF-associated pathology.

\section{Sweat gland}

Excessive secretion of sodium chloride by sweat glands is characteristic of CF; in fact, the majority of patients diagnosed with CF have sweat chloride levels exceeding $60 \mathrm{mmol} \cdot \mathrm{L}^{-1}$ [20]. In healthy individuals, CFTR protein is responsible for reabsorption of chloride (and subsequently sodium) in the reabsorptive duct of the sweat gland. Absence or dysfunction of CFTR impedes this process, giving rise to hypertonic beads of sweat as seen in CF [20,21].

To investigate the relationship between CFTR functionality and sweat chloride concentration, ROWE et al. [22] evaluated sweat chloride levels in CF patients as a function of predicted CFTR activity. CFTR activity was based on previous reports of the relationship between CFTR genotype and phenotype and on in vitro investigations (fig. 2). The authors assumed that normal individuals have $100 \%$ CFTR activity whereas healthy carriers have CFTR activity of $50 \%$. In this study, normal adults without CFTR mutations had a mean sweat chloride concentration of $\sim 20 \mathrm{mmol} \cdot \mathrm{L}^{-1}$. Carriers, with a single CFTR mutation, showed a small increase in sweat chloride levels compared to normal subjects, with mean levels of $\sim 26 \mathrm{mmol} \cdot \mathrm{L}^{-1}$. CF patients with one severe and one mild CFTR mutation were reported to have an average sweat chloride of $\sim 80 \mathrm{mmol} \cdot \mathrm{L}^{-1}$. Those with two severe mutations had the most severe sweat chloride abnormalities with an average in excess of $100 \mathrm{mmol} \cdot \mathrm{L}^{-1}$. This study demonstrated a clear correlation between sweat chloride levels and CFTR functionality. However, it should be noted that there

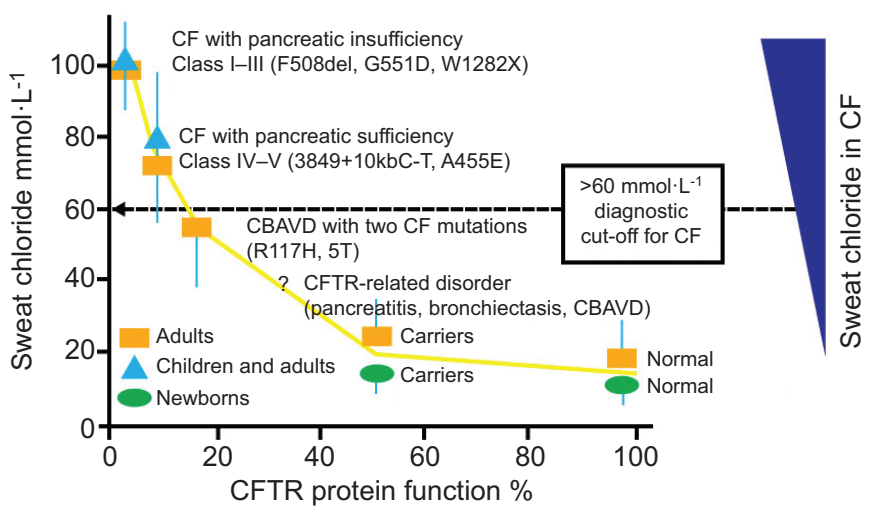

FIGURE 2. Correlation of sweat chloride levels with cystic fibrosis transmembrane conductance regulator (CFTR) protein expression and function. In cystic fibrosis (CF) patients with Class I-III CFTR mutations, absent or minimal CFTR function results in exocrine pancreatic insufficiency and highly elevated levels of sweat chloride concentration. Residual CFTR function in CF patients with Class IV$\checkmark$ CFTR mutations is associated with exocrine pancreatic sufficiency and sweat chloride levels above or below the diagnostic cut-off of $60 \mathrm{mmol} \cdot \mathrm{L}^{-1}$. CFTR-related disorders are non-CF conditions with some CFTR dysfunction, usually presenting with intermediate or normal sweat chloride values and CFTR mutations that are known to affect mRNA production, although to a lesser degree than CF-causing CFTR mutations [23]. CBAVD: congenital bilateral absence of vas deferens, Modified from [22] with permission from the publisher.

are several potential drawbacks of the sweat test, including reports of false-negative or false-positive results [24].

\section{Pancreas and GI tract}

Pancreatic insufficiency is thought to occur in $90-95 \%$ of CF patients. Defective secretion of digestive enzymes and resulting fat malabsorption in the gastrointestinal system has several consequences including steatorrhea and failure to thrive. In addition, ionic imbalance in the biliary tract may lead to increased risk of gall stone and hepatobiliary disease [2, 19]. Pancreatic abnormalities also can cause CF-related diabetes, which has a prevalence of $\sim 25 \%$ in CF patients aged $>25 \mathrm{yrs}$ [8].

\section{Reproductive system}

Around $99 \%$ of adult males with CF have congenital bilateral absence of the vas deferens, which is a developmental defect that blocks the transport of spermatozoa from the testes or epididymis to the vas deferens resulting in azoospermia $[9,25]$. Generally speaking, infertility in females with $\mathrm{CF}$ has a less serious outcome than in males and is thought to be due to thickening of cervical mucus [26].

\section{Airways}

The most significant clinical manifestations of $\mathrm{CF}$ are in the lung and airways. In bronchial tissues, CFTR protein is expressed in the submucosal glands and the apical surface of ciliated epithelial cells $[27,28]$, and has been shown to play a critical role in epithelial wound repair [29]. Normally, effective mucociliary transport is facilitated by sufficient hydration of the airway surface liquid (ASL); an important periciliary and mucus layer that lines the airway tract [30,31]. ASL hydration is achieved through establishment of an osmotic gradient by a predominant efflux of chloride ions through CFTR channels, coupled with a moderate influx of sodium ions through epithelial 
sodium channels in the apical membrane. Absence or dysfunction of CFTR leads to a lack of chloride efflux and unregulated hyperabsorption of sodium ions. This imbalance of the osmotic gradient causes ASL dehydration, increased mucoviscosity and impaired mucociliary transport [30, 32]. Without intervention, this situation gradually deteriorates causing plugging of small airways, bacterial infection, chronic local inflammation and eventually bronchiectasis [9, 19, 21]. Furthermore, repeated pulmonary exacerbations in CF have been shown to contribute to an accelerated decline in lung function [33].

\section{DEVELOPMENT OF CF DISEASE-MODIFYING THERAPIES}

Disease-modifying strategies can be classified into two main categories: agents that target the genetic mutation (i.e. gene therapy) and approaches that address the associated protein defect, such as CFTR modulators.

\section{Gene therapy}

CF gene therapy aims at delivering copies of the coding sequence of wild-type (i.e. normal) CFTR DNA to target cells. This can be achieved by using either viral or non-viral delivery methods [34]. The former uses recombinant viral vectors, which have their viral DNA removed to act as a vehicle for delivery of therapeutic DNA to the host cell. The viral vector binds to the host cell to introduce the coding sequence of wildtype CFTR, which ideally should be persistently transcribed into mRNA, or even be integrated into the cell genome. To date, adenovirus, adeno-associated viruses, negative strand RNA viruses and lentivirus have been used as viral delivery systems for CFTR [34].

Non-viral gene therapy involves the delivery of naked DNA or DNA complexes into the cell by vectors other than viruses. Non-viral vectors under investigation include cationic lipid GL67A, DNA nanoparticles (DNA complexed with peptides) and self-assembling polyethylenimine complexes $[9,35,36]$. DAVIES and ALTON [35] have reported that CFTR gene transfer can be reproducibly achieved with non-viral vectors. The authors commented that the overall duration of expression was relatively short and side-effects were noted with both viral and non-viral techniques.

Since the 1990s, research into gene therapy has not provided clear evidence of how these findings might relate to tangible benefits for clinical practice and currently no gene therapies have been approved for use $[9,35]$. In fact, in recent years research into gene therapy has become increasingly limited, largely due to the evidence that gene transfer is more challenging than originally thought; trials in this area are expensive to conduct and research to date suggested limited efficacy of gene transfer vectors [34]. Nevertheless, the UK CF Gene Therapy Consortium recently embarked on a gene therapy programme. This research is aimed at assessing if repeated administration of the non-viral gene transfer agent, cationic lipid GL67, can improve lung disease in CF [34].

\section{CFTR modulators}

The last 10 yrs have seen promising results from research into the management of CF using small molecules called CFTR modulators. This research relies on extensive knowledge of CFTR mutation classes and focuses on targeting certain mutations with specific molecules [9]. These very specialised and individualised molecules are a typical example of personalised medicine in practice, where only those individuals with a specific mutation can be treated with the target drug [37]. CFTR modulators can be categorised into three main classes of agents: potentiators, correctors and premature stop codon suppressors or read-through agents. They are invariably identified using high-throughput screening, an automated approach which allows the rapid screening of thousands of small molecules. This technique offers a swifter means of identifying small molecules than the traditional method of testing each component individually using a cell culture system [9]. As a result, several candidate molecules are now undergoing clinical trials, a few of which are discussed below.

\section{Read-through agents}

Premature stop mutations, or nonsense mutations, account for between $5 \%$ and $10 \%$ of all CFTR mutations, with high regional variations $[3,38]$. Class I CFTR nonsense mutations can be targeted using premature stop codon suppressors, more commonly referred to as read-through agents, which "force" read-through of premature stop codons during translation of the CFTR protein. This results in the production of a full-length CFTR protein, which may then be trafficked to the cell surface.

Several examples of read-through agents exist. For example, treating cells in vitro with low doses of an aminoglycoside antibiotic demonstrated that two CFTR-associated stop mutations could be suppressed [38]. The most commonly investigated antibiotic, gentamicin, acts by allowing an amino acid to be incorporated in place of a stop codon so that translation continues as normal. In one study, the effectiveness of topically applied nasal gentamicin was evaluated in $24 \mathrm{CF}$ patients. In this study, 19 CF patients were either homozygous $(n=11)$ or heterozygous $(n=8)$ for CFTR stop mutations and a total of five patients were homozygous for the Class II mutation F508del [39]. Gentamicin was shown to significantly reduce the nasal potential difference (a measure of CFTR function) in those patients with stop mutations (from basal potential difference $45 \pm 8$ to $34 \pm 11 \mathrm{mV}, \mathrm{p}=0.005$ ), whereas no significant difference was seen in patients homozygous for F508del [39]. Positive effects were also demonstrated by SERMET-GAUDELUS et al. [40], although no effect was noted in another study [41]. While research into other aminoglycosides is ongoing and techniques to limit potential toxicities are being investigated [42], current literature suggests that any clinical benefit is limited by the need for intravenous or intramuscular administration, as well as its lack of potency and potential renal toxicity [43].

Ataluren, formerly known as PTC124, is potentially a more promising read-through agent than gentamicin. Using a stable cell line, ataluren was shown to be a 4 - to 15 -fold more potent read-through agent relative to controls [43]. The agent is available in an easy-to-use formulation and is currently undergoing clinical trials. Initial clinical studies in healthy adults did not raise any safety concerns and analysis of subjects' peripheral blood mononuclear cells showed no aberrant elongation of CFTR protein [44]. This could have occurred due to nonspecific ribosomal read-through of normal stop codons. In a study of paediatric patients ataluren significantly increased the proportion of nasal epithelial cells expressing full-length CFTR protein in the apical membrane (overall mean change $+17.0 \%$; $<<0.01$ ) [45]. 
The authors concluded that ataluren can induce functional CFTR production and is well tolerated. A phase II study in 19 adult patients with CF, having at least one CFTR nonsense mutation allele and an abnormal nasal total chloride transport, showed that ataluren three times daily improved total chloride transport (combined mean change of $-5.4 \mathrm{mV}, \mathrm{p}<0.001$ ), with an overall on-treatment response rate of $61 \%(p<0.001)$. There was also a nonsignificant trend towards improvement in forced expiratory volume in $1 \mathrm{~s}$ (FEV1) and a 23\% reduction in cough $(p=0.006)$ by the end of the study (day 84) [46].

\section{CFTR correctors}

CFTR correctors are designed to increase the amount of functional CFTR protein delivered to the cell surface. Correctors have been used to target Class II mutations such as F508del [47, 48]. One such molecule, VX-809, is currently under clinical investigation. In vitro studies demonstrated VX-809 as an efficacious and selective CFTR corrector suitable for advancement into clinical studies [49]. In a phase Ilb study, VX-809 was investigated in adults who were homozygous for the F508del-CFTR mutation [50]. Sweat chloride levels in VX-809-treated CF subjects were reduced in a dose-dependent manner $(p=0.0013)$. Overall, the effect, although modest, was statistically significant. No significant differences were seen between control and treatment arms in measures of lung function (FEV1, forced vital capacity or forced expiratory flow at $25-75 \%$ of forced vital capacity) or in CFTR-dependent nasal potential difference parameters. The authors concluded that further data are required to investigate any impact in the respiratory tract and to determine possible benefits for clinical practice. In addition to VX-809, various other CFTR correctors have been identified, some of which are under further preclinical investigation $[11,51]$.

\section{CFTR potentiators}

CFTR potentiators increase the open probability of CFTR channels that have gating (Class III) or conductance (Class IV) mutations [9]. There is additional evidence from in vitro studies that CFTR potentiators may also enhance the open probability of CFTR channels with Class II mutations, such as F508del [52]. Nevertheless, in order for F508del potentiation to occur in this system, the recombinantly expressed channel must already be located on the cell membrane. Thus, CFTR potentiators alone could not be used to treat Class I or II mutations, which are characterised by an absence or lack or synthesised CFTR protein.

Ivacaftor, formerly known as VX-770, is a CFTR potentiator that specifically targets the Class III mutation, G551D. The compound was initially identified using high-throughput screening from a library of 228,000 agents and subsequently validated using single channel patch-clamp measurements in Fischer Rat Thyroid cells expressing G551D-CFTR [52]. The authors reported that $10 \mu \mathrm{M}$ ivacaftor increased the open probability of G551D-CFTR channels by $\approx 6$-fold $(p<0.05)$. These findings were validated in primary cultures of human bronchial epithelial (HBE) cells isolated from the bronchi of G551D-CF patients. HBE primary cell cultures are reported to accurately represent the native pathological environment of $\mathrm{CF}$ lung disease and can be used to reliably characterise the pharmacology of CFTR modulators [53]. In HBE cells with the G551D/F508del and F508del/F508del genotypes, ivacaftor was shown to potentiate CFTR-mediated chloride secretion, with the greatest effect seen in G551D/F508del cells (50\% of wildtype CFTR activity) (fig. 3a). Moreover, the ciliary beat frequency $(\mathrm{CBF})$ increased to levels observed in non-CF HBE (fig. 3b). This can be explained by an accompanying dosedependent increase in the volume of ASL (fig. 3c and d) to approximately half of that observed in non-CF HBE [52]. ASL and $\mathrm{CBF}$ are markers of lung function that are routinely used in $\mathrm{CF}$ research to help translate in vitro findings in primary HBE cultures into patient-related outcomes in clinical practice. Taken together, these preclinical results suggest that ivacaftor specifically targets and repairs the underlying gating defect caused by the G551D mutation. Based on this evidence clinical trials were commenced in CF patients carrying at least one copy of the G551D-CFTR mutation, the results of which are discussed in the article by SERMET-GAUDELUS [55] in this issue of the European Respiratory Review.

\section{ADVANCING CF DRUG DISCOVERY USING SENSITIVE SURROGATE BIOMARKERS}

Recent CFTR modulator clinical development programmes have demonstrated how in vitro studies using cell culture systems can provide a sound basis for translation of pre-clinical investigations into clinical trials. It remains to be seen whether recent pig and ferret $\mathrm{CF}$ models provide a more accurate prediction of human response to therapeutic intervention and if this can be correlated with clinical biomarkers [56, 57].

An essential aspect of delivering personalised medicine is the identification of biomarkers that accurately assess the extent of disease whilst predicting and measuring response to treatment. In $\mathrm{CF}$, the sweat test and nasal potential difference test have been widely used to assess CFTR activity in CF clinical trials. In fact the sweat test, which is used to measure skin sweat chloride levels, is the established gold standard for diagnosing CF $[58,59]$. Both the sweat test and nasal potential difference have been effectively used as end-points to measure therapeutic efficacy in clinical trials of ivacaftor, VX-809, and ataluren. Nevertheless, test variability and organ-specific treatment responsiveness remain challenging issues [60].

\section{Intestinal current measurement: a robust biomarker for therapeutic development}

CFTR protein is expressed in the human intestine, with some reports suggesting that the channel is found at higher levels in rectal tissue [61]. The degree of CFTR activity in the intestine can be measured using an electrophysiological test: the intestinal current measurement (ICM) [62]. Studies have shown that ICM correlates with CFTR genotype and phenotype [63], and diagnostic sensitivity in difficult CF diagnosis remarkably reaches $100 \%$ [64], making this tool also a potentially useful CF biomarker. Notably, defects in CFTR-mediated chloride transport correlated with $\mathrm{CF}$ disease severity, suggesting that ICM accurately reflects the level of CFTR functionality. Moreover, pancreatic insufficient patients with Class I-III mutations had absent or minimal chloride secretion; a high amount of residual function was observed in pancreatic sufficient CF patients with Class IV-V mutations and normal CFTR function was noted in heterozygotes and healthy controls [64].

Building on this evidence, the suitability of ICM as a tool for preclinical assessment of CFTR modulators was recently evaluated using human rectal suction biopsies from CF outpatients [65]. 


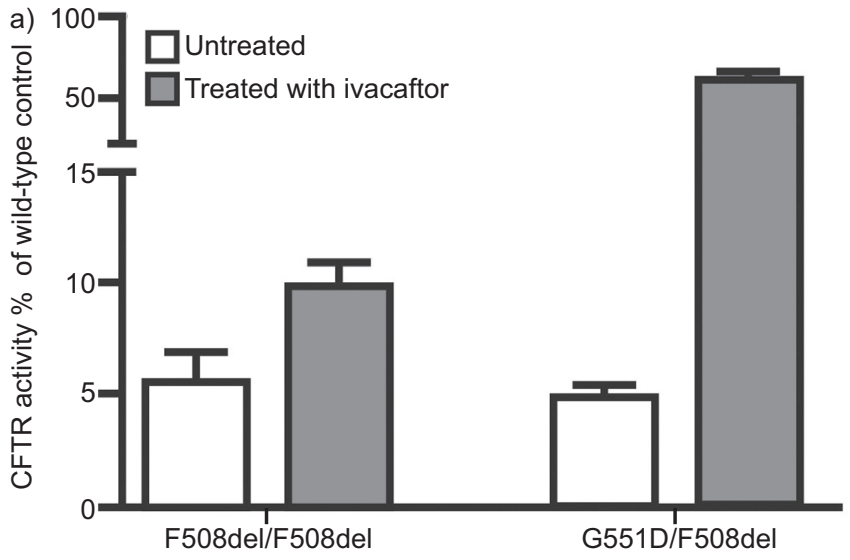

c)
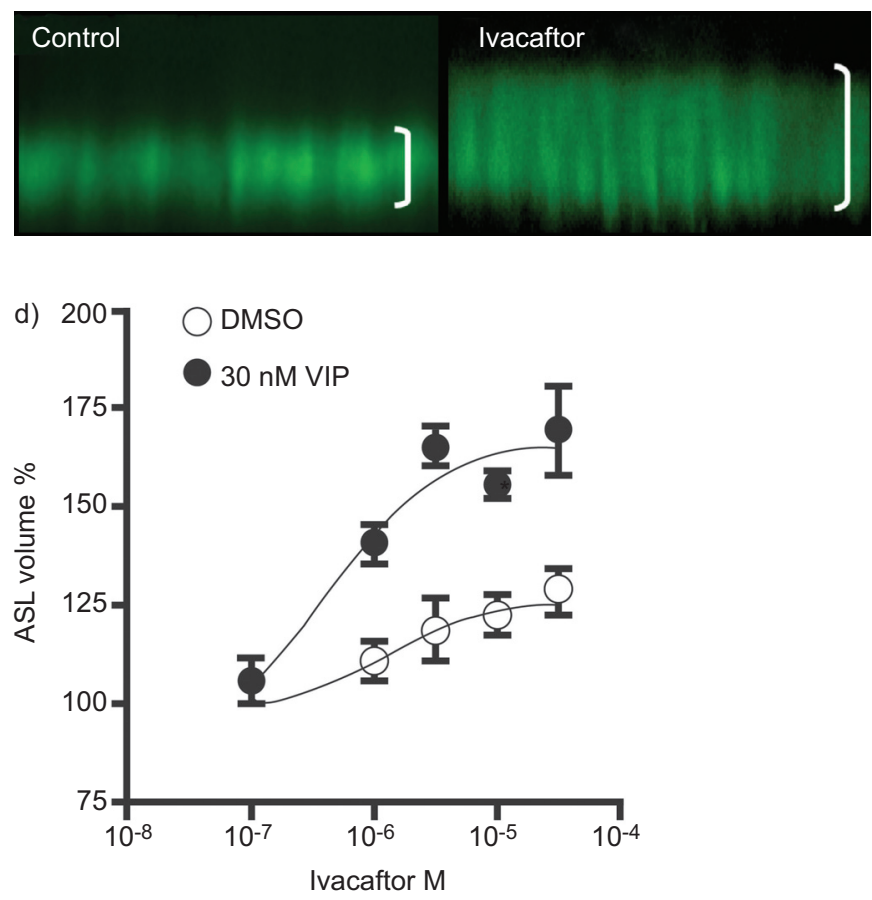

These investigations strengthened increasing evidence that the human rectal ICM test is repeatable, reproducible and responsive to ex vivo treatment with CFTR modulators. As a useful addition to in vitro studies in HBE cultures, the technique might be helpful for prediction of CFTR mutationspecific in vivo drug effects.

As few limitations of this test have been reported thus far, it is possible that an increasing number of CF centres will rely on ICM tests in the future. Standardisation for multicentre ICM use in clinical trials has been recently performed [66].

\section{CONCLUSIONS}

CF is characterised by a clear link between the genetics of CFTR dysfunction and disease pathophysiology. Understanding this link has led to the development of CFTR modulators that target specific CFTR genotypes, based on the mechanism of protein dysfunction. This novel class of agents were first identified by high-throughput screening before being validated and

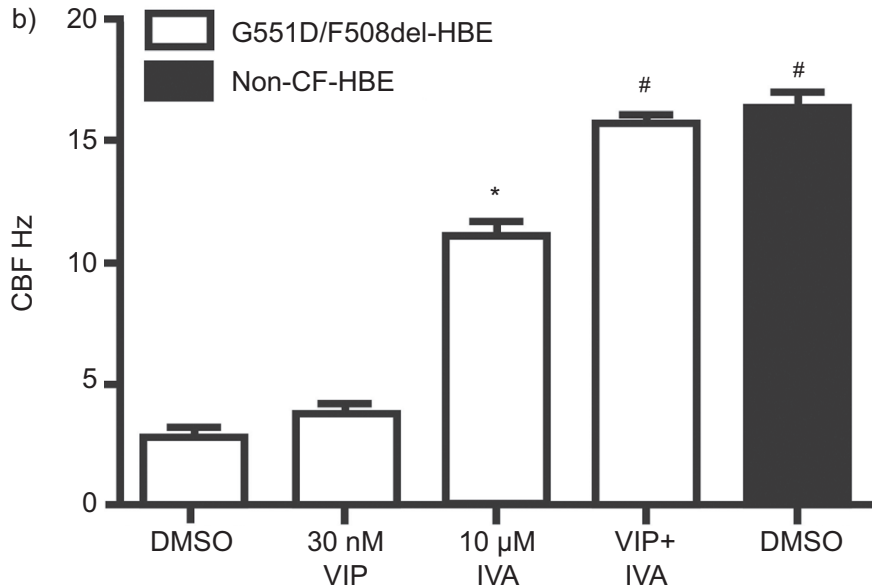

FIGURE 3. Ivacaftor restores G551D-cystic fibrosis transmembrane conductance regulator (CFTR) function in human bronchial epithelial (HBE) primary cell cultures. a) CFTR activity was evaluated by measuring CFTR-dependent chloride transport (short-circuit current in voltage-clamp mode) relative to wild-type $(n=16)$. Ivacaftor increased the activity of G551D/F508del-CFTR to $~ 50 \%$ of wild-type (personal communication; F. van Goor, Vertex Pharmaceuticals Inc., Cambridge, MA, USA). Modified from [54]. b) Mean ciliary beat frequency (CBF) after 5 days of treatment with dimethyl sulfoxide (DMSO), $30 \mathrm{nM}$ vasoactive intestinal peptide (VIP) (a naturally occuring CFTR activator), $10 \mu \mathrm{M}$ ivacaftor (IVA), or $30 \mathrm{nM}$ VIP+10 $\mu \mathrm{M}$ IVA ( \pm SEM; $n=6$ ). Taken together, the results show that IVA (either alone or in combination with VIP) caused a significant increase in CBF with respect to vehicle control (DMSO). Modified from [52]. *: $p<0.05$, significant difference between DMSO and G551D/F508del-HBE; ${ }^{*}:$ p <0.05, significant difference between DMSO and IVA alone. c) Representative confocal microscopy image of G551D/F508del-HBE cultures treated with vehicle control or ivacaftor. The results show a clear ivacaftordependent increase in the depth of airway surface liquid (ASL) relative to control (personal communication; F. van Goor, Vertex Pharmaceuticals Inc., Cambridge, MA, USA). Modified from [52]. d) Dose-response curve of the change in ASL volume in G551D/F508del-HBE after application of ivacaftor at the indicated concentrations $(n=3-9)$. Reproduced from [52].

optimised using in vitro culture systems. Promising candidates were subsequently evaluated in clinical trials. One such modulator, the CFTR potentiator ivacaftor, is a first-in-class agent to be approved as an oral therapy for CF patients with the G551D-CFTR mutation and is a pertinent example of personalised medicine. The availability of more sensitive surrogate biomarkers of CF disease, such as intestinal current measurements, may serve to streamline the development of future CFTR modulators.

\section{STATEMENT OF INTEREST}

N. Derichs received speaker honorarium from Vertex Pharmaceuticals Inc. for participation in a symposium and served as a consultant for Vertex Inc. at educational activities and advisory boards.

\section{ACKNOWLEDGEMENTS}

I would like to thank Facilitate Ltd (Brighton, UK), funded by Vertex Pharmaceuticals Inc., USA, for editorial assistance in the production of this manuscript. 


\section{REFERENCES}

1 Andersen DH. Cystic fibrosis of the pancreas and its relation to celiac disease. Am J Dis Childhood 1938; 56: 344-399.

2 Davis PB, Drumm M, Konstan MW. Cystic fibrosis. Am J Respir Crit Care Med 1996; 154: 1229-1256.

3 Kerem B, Rommens JM, Buchanan JA, et al. Identification of the cystic fibrosis gene: genetic analysis. Science 1989; 245: 1073-1080.

4 Riordan JR, Rommens JM, Kerem B, et al. Identification of the cystic fibrosis gene: cloning and characterization of complementary DNA. Science 1989; 245: 1066-1073.

5 Rommens JM, Iannuzzi MC, Kerem B, et al. Identification of the cystic fibrosis gene: chromosome walking and jumping. Science 1989; 245: 1059-1065.

6 Elborn SJ. Personalised medicine for cystic fibrosis: treating the basic defect. Eur Respir Rev 2013; 22: 3-5.

7 World Health Organization. The molecular genetic epidemiology of cystic fibrosis. Report of a joint meeting of WHO/ECFTN/ ICF(M)A/ECFS. WHO/HGN/CF/WG/04.02. Geneva, WHO/ $\mathrm{HGN} / \mathrm{CF} / \mathrm{WG}, 2004$

8 Cystic Fibrosis Foundation. Patient Registry Annual Data Report. Bethesda, CF Foundation, 2011.

9 Rogan MP, Stoltz DA, Hornick DB. Cystic fibrosis transmembrane conductance regulator intracellular processing, trafficking, and opportunities for mutation-specific treatment. Chest 2011; 139: 1480-1490.

10 Clinical and Functional Translation of CFTR (CFTR2) www.cftr2. org Date last accessed: December 16, 2012.

11 Clancy JP, Jain M. Personalized medicine in cystic fibrosis: dawning of a new era. Am J Respir Crit Care Med 2012; 186: 593-597.

12 Castellani C, Cuppens H, Macek M Jr, et al. Consensus on the use and interpretation of cystic fibrosis mutation analysis in clinical practice. J Cyst Fibros 2008; 7: 179-196.

13 Worldwide survey of the delta F508 mutation - report from the cystic fibrosis genetic analysis consortium. Am J Hum Genet 1990; 47: 354-359.

14 Rowntree RK, Harris A. The phenotypic consequences of CFTR mutations. Ann Hum Genet 2003; 67: 471-485.

15 Zielenski J. Genotype and phenotype in cystic fibrosis. Respiration 2000; 67: 117-133.

16 MacDonald KD, McKenzie KR, Zeitlin PL. Cystic fibrosis transmembrane regulator protein mutations: "class" opportunity for novel drug innovation. Paediatric drugs 2007; 9: 1-10.

17 Kirk KL, Wang W. A unified view of cystic fibrosis transmembrane conductance regulator (CFTR) gating: combining the allosterism of a ligand-gated channel with the enzymatic activity of an ATP-binding cassette (ABC) transporter. J Biol Chem 2011; 286: 12813-12819.

18 Gelman MS, Kopito RR. Cystic fibrosis: premature degradation of mutant proteins as a molecular disease mechanism. Methods $\mathrm{Mol}$ Biol 2003; 232: 27-37.

19 Flume PA, Van Devanter DR. State of progress in treating cystic fibrosis respiratory disease. BMC medicine 2012; 10: 88.

20 Quinton PM. Cystic fibrosis: lessons from the sweat gland. Physiology 2007; 22: 212-225.

21 Rowe SM, Miller S, Sorscher EJ. Cystic fibrosis. N Engl J Med 2005; 352: 1992-2001.

22 Rowe SM, Accurso F, Clancy JP. Detection of cystic fibrosis transmembrane conductance regulator activity in early-phase clinical trials. Proc Am Thorac Soc 2007; 4: 387-398.

23 Bombieri C, Claustres M, De Boeck K, et al. Recommendations for the classification of diseases as CFTR-related disorders. J Cyst Fibros 2011; 10: Suppl. 2, S86-S102.

24 Mishra A, Greaves R, Massie J. The relevance of sweat testing for the diagnosis of cystic fibrosis in the genomic era. Clin Biochem Rev 2005; 26: 135-153.

25 Radpour R, Gourabi H, Dizaj AV, et al. Genetic investigations of CFTR mutations in congenital absence of vas deferens, uterus, and vagina as a cause of infertility. J Androl 2008; 29: 506-513.
26 Chan HC, Ruan YC, He Q, et al. The cystic fibrosis transmembrane conductance regulator in reproductive health and disease. J Physiol 2009; 587: 2187-2195.

27 Engelhardt JF, Yankaskas JR, Ernst SA, et al. Submucosal glands are the predominant site of CFTR expression in the human bronchus. Nat Genet 1992; 2: 240-248.

28 Kreda SM, Mall M, Mengos A, et al. Characterization of wild-type and deltaF508 cystic fibrosis transmembrane regulator in human respiratory epithelia. Mol Biol Cell 2005; 16: 2154-2167.

29 Trinh NT, Bardou O, Prive A, et al. Improvement of defective cystic fibrosis airway epithelial wound repair after CFTR rescue. Eur Respir J 2012; 40: 1390-1400.

30 Boucher RC. New concepts of the pathogenesis of cystic fibrosis lung disease. Eur Respir J 2004; 23: 146-158.

31 Goralski JL, Boucher RC, Button B. Osmolytes and ion transport modulators: new strategies for airway surface rehydration. Curr Opin Pharmacol 2010; 10: 294-299.

32 Derichs N, Jin BJ, Song Y, et al. Hyperviscous airway periciliary and mucous liquid layers in cystic fibrosis measured by confocal fluorescence photobleaching. FASEB J 2011; 25: 2325-2332.

33 Waters V, Stanojevic S, Atenafu EG, et al. Effect of pulmonary exacerbations on long-term lung function decline in cystic fibrosis. Eur Respir J 2012; 40: 61-66.

34 Griesenbach U, Alton EW. Progress in gene and cell therapy for cystic fibrosis lung disease. Cur Pharm Des 2012; 18: 642-662.

35 Davies JC, Alton EW. Gene therapy for cystic fibrosis. Proc Am Thorac Soc 2010; 7: 408-414

36 Rosenecker J, Huth S, Rudolph C. Gene therapy for cystic fibrosis lung disease: current status and future perspectives. Curr Opin Mol Ther 2006; 8: 439-445.

37 Pettit RS. Cystic fibrosis transmembrane conductance regulatormodifying medications: the future of cystic fibrosis treatment. Ann Pharmacother 2012; 46: 1065-1075.

38 Howard M, Frizzell RA, Bedwell DM. Aminoglycoside antibiotics restore CFTR function by overcoming premature stop mutations. Nat Med 1996; 2: 467-469.

39 Wilschanski M, Yahav Y, Yaacov Y, et al. Gentamicin-induced correction of CFTR function in patients with cystic fibrosis and CFTR stop mutations. N Engl J Med 2003; 349: 1433-1441.

40 Sermet-Gaudelus I, Renouil M, Fajac A, et al. In vitro prediction of stop-codon suppression by intravenous gentamicin in patients with cystic fibrosis: a pilot study. BMC Med 2007; 5: 5.

41 Clancy JP, Rowe SM, Bebok Z, et al. No detectable improvements in cystic fibrosis transmembrane conductance regulator by nasal aminoglycosides in patients with cystic fibrosis with stop mutations. Am J Respir Cell Mol Biol 2007; 37: 57-66.

42 Prayle A, Smyth AR. Aminoglycoside use in cystic fibrosis: therapeutic strategies and toxicity. Curr Opin Pulm Med 2010; 16 604-610.

43 Welch EM, Barton ER, Zhuo J, et al. PTC124 targets genetic disorders caused by nonsense mutations. Nature 2007; 447: 87-91.

44 Hirawat S, Welch EM, Elfring GL, et al. Safety, tolerability, and pharmacokinetics of PTC124, a nonaminoglycoside nonsense mutation suppressor, following single- and multiple-dose administration to healthy male and female adult volunteers. I Clin Pharmacol 2007; 47: 430-444.

45 Sermet-Gaudelus I, Boeck KD, Casimir GJ, et al. Ataluren (PTC124) induces cystic fibrosis transmembrane conductance regulator protein expression and activity in children with nonsense mutation cystic fibrosis. Am J Respir Crit Care Med 2010; 182: 1262-1272.

46 Wilschanski M, Miller LL, Shoseyov D, et al. Chronic ataluren (PTC124) treatment of nonsense mutation cystic fibrosis. Eur Respir J 2011; 38: 59-69.

47 Pedemonte N, Lukacs GL, Du K, et al. Small-molecule correctors of defective DeltaF508-CFTR cellular processing identified by highthroughput screening. J Clin Invest 2005; 115: 2564-2571. 
48 Van Goor F, Straley KS, Cao D, et al. Rescue of deltaF508-CFTR trafficking and gating in human cystic fibrosis airway primary cultures by small molecules. Am J Physiol Lung Cell Mol Physiol 2006; 290: L1117-L1130.

49 Van Goor F, Hadida S, Grootenhuis PD, et al. Correction of the F508del-CFTR protein processing defect in vitro by the investigational drug VX-809. Proc Natl Acad Sci USA 2011; 108: 18843-18848.

50 Clancy JP, Rowe SM, Accurso FJ, et al. Results of a phase IIa study of VX-809, an investigational CFTR corrector compound, in subjects with cystic fibrosis homozygous for the F508del-CFTR mutation. Thorax 2012; 67: 12-18.

51 Cai ZW, Liu J, Li HY, et al. Targeting F508del-CFTR to develop rational new therapies for cystic fibrosis. Acta Pharmacologica Sinica 2011; 32: 693-701.

52 Van Goor F, Hadida S, Grootenhuis PD, et al. Rescue of CF airway epithelial cell function in vitro by a CFTR potentiator, VX-770. Proc Natl Acad Sci USA 2009; 106: 18825-18830.

53 Neuberger T, Burton B, Clark H, et al. Use of primary cultures of human bronchial epithelial cells isolated from cystic fibrosis patients for the pre-clinical testing of CFTR modulators. Methods Mol Biol 2011; 741: 39-54.

$54 \mathrm{Yu} \mathrm{H}$, Burton B, Huang C, et al. Ivacaftor potentiation of multiple CFTR channels with gating mutations. J Cyst Fibros 2012; 11: 237-245.

55 Sermet-Gaudelus I. Ivacaftor treatment in patients with cystic fibrosis and the G551D-CFTR mutation. Eur Respir Rev 2013; 22: 66-71.

56 Fisher JT, Zhang Y, Engelhardt JF. Comparative biology of cystic fibrosis animal models. Methods Mol Biol 2011; 742: 311-334.

57 Keiser NW, Engelhardt JF. New animal models of cystic fibrosis: what are they teaching us? Curr Opin Pulm Med 2011; 17: 478-483.
58 Gibson LE, Cooke RE. A test for concentration of electrolytes in sweat in cystic fibrosis of the pancreas utilizing pilocarpine by iontophoresis. Pediatrics 1959; 23: 545-549.

59 De Boeck K, Wilschanski M, Castellani C, et al. Cystic fibrosis: terminology and diagnostic algorithms. Thorax 2006; 61: 627-635.

60 De Boeck K, Kent L, Davies J, et al. CFTR biomarkers: time for promotion to surrogate end-point? Eur Respir J 2013; 41: 203-216.

61 Doucet L, Mendes F, Montier T, et al. Applicability of different antibodies for the immunohistochemical localization of CFTR in respiratory and intestinal tissues of human and murine origin. J Histochem Cytochem 2003; 51: 1191-1199.

62 Veeze HJ, Sinaasappel M, Bijman J, et al. Ion transport abnormalities in rectal suction biopsies from children with cystic fibrosis. Gastroenterology 1991; 101: 398-403.

63 Hirtz S, Gonska T, Seydewitz HH, et al. CFTR $\mathrm{Cl}^{-}$channel function in native human colon correlates with the genotype and phenotype in cystic fibrosis. Gastroenterology 2004; 127: 1085-1095.

64 Derichs N, Sanz J, Von Kanel $\mathrm{T}$, et al. Intestinal current measurement for diagnostic classification of patients with questionable cystic fibrosis: validation and reference data. Thorax 2010; 65: 594-599.

65 Derichs N, Knoll J, Hyde R, et al. Preclinical evaluation of CFTR modulators in ex vivo human rectal tissue. Pediatr Pulmonol 2009; 44: 292.

66 European Cystic Fibrosis Society. ECFS Diagnostic Network Working Group. www.ecfs.eu/ecfs_dnwg Date last updated: November 13, 2012. Date last accessed: December 16, 2012. 